\title{
Utilização de macroinvertebrados bentônicos como bioindicadores em córrego urbano de Conceição do Araguaia-PA
}

\author{
Use of benthic macroinvertebrates as bioindicators in an \\ urban stream in Conceição do Araguaia-PA
}

Maria Elisa Ferreira de Queiroz ${ }^{\mathrm{a}}$

Alexandre Luiz Schäffer ${ }^{b}$

Ana Carolyna Aparecida Silva Villelac

Danilo Epaminondas Martins e Martins ${ }^{d}$

Paulo Henrique Teles da Silva ${ }^{e}$

${ }^{a}$ Doutoranda no Programa de Ciências Ambientais da Universidade Federal do Pará, Belém, PA, Brasil.

End. Eletrônico: queirozluna@gmail.com

${ }^{b}$ Mestrando em Ambiente e Tecnologias Sustentáveis pela Universidade Federal da Fronteira Sul, Cerro Largo, RS, Brasil. End. Eletrônico: alexandreluiz1992@hotmail.com

'Mestranda em Engenharia Agrícola pela Universidade Estadual do Oeste do Paraná, Cascavel, PR, Brasil. End. Eletrônico: carol-ly@hotmail.com

${ }^{d}$ Mestrando em Ambiente e Tecnologias Sustentáveis pela Universidade Federal da Fronteira Sul, Cerro Largo, RS, Brasil. End. Eletrônico: epaminondasmartins@hotmail.com

'Mestrando em Biodiversidade, Ecologia e Conservação pela Universidade Federal do Tocantins, Porto Nacional, TO, Brasil. End. Eletrônico: noturno00@hotmail.com

doi:10.18472/SustDeb.v9n3.2018.18378

Recebido em 24.03.2018

Aceito em 04.09.2018

ARTIGO - VARIA

\section{RESUMO}

Os macroinvertebrados bentônicos têm sido utilizados para avaliar a qualidade da água de rios e lagos, visto que sua distribuição e ocorrência relacionam-se a diferentes fatores ambientais. Desse modo, objetivou-se avaliar as condições ambientais e de qualidade da água de um córrego urbano no estado do Pará (Brasil), por meio da captura desses organismos e posterior classificação pelo índice Biological Monitoring Working Party Score System (BMWP). Esse método se baseia na tolerância dos organismos ao impacto no meio, gerando pontuações àqueles presentes no ambiente e assim constituindo um índice da qualidade da água no local. Como resultados, os 2.288 indivíduos coletados ( 8 ordens e 23 famílias), pertencentes aos filos Mollusca, Anelida e Arthropoda, apresentaram baixa pontuação e a maioria dos pontos amostrados (73\%) foram classificados como contaminados, refletindo as consequências oriundas do crescimento urbano desordenado e das precárias condições de saneamento básico que o estado apresenta.

Palavras-chave: Biomonitoramento; Índice BMWP; Emerêncio; Qualidade ambiental. 


\begin{abstract}
The benthic macroinvertebrates have been used to evaluate the water quality of rivers and lakes, since their distribution and occurrence are related to different environmental factors. The aim of this study is to evaluate the environmental and water quality conditions of an urban stream in the state of Pará (Brazil), by capturing these organisms and later classifying them by the Biological Monitoring Working Party Score System (BMWP) index. This method is based on the tolerance of the organisms to the impact with the environment, generating scores to those present in the environment and by that constituting an index of water quality in the place. As a result, the 2,288 individuals collected (8 orders and 23 families), belonging to the Mollusca, Anelida and Arthropoda phyla, presented low scores and most of the points sampled (73\%) were classified as contaminated, reflecting the consequences of disordered urban growth and the precarious conditions of basic sanitation that the state presents.
\end{abstract}

Keywords: Biomonitoring; BMWP Index; Emerêncio; Environmental quality.

\title{
1 INTRODUÇÃO
}

Em áreas com altas densidades populacionais, as atividades antrópicas vêm afetando diretamente os ecossistemas aquáticos. Os principais problemas localizam-se onde os cursos de água recebem o aporte de esgotos in natura, sedimentos e uma alta carga de resíduos sólidos, resultando na alteração das características físicas, químicas e biológicas (SILVA, 2007).

Para avaliar essas alterações, normalmente realiza-se o monitoramento da qualidade da água com análises de $\mathrm{pH}$, condutividade, temperatura, Demanda Bioquímica de Oxigênio (DBO), Demanda Química de Oxigênio (DQO), fosfato, nitrato e nitrito, entre outras variáveis que permitem inferir sobre as condições atuais do ambiente, entretanto, além do custo elevado dessas análises, são necessários longos períodos de amostragem para afirmar as causas e os efeitos de uma possível contaminação (LIMA et al., 2018). Técnicas que envolvem o biomonitoramento podem se tornar uma alternativa viável para avaliar tais alterações.

O uso sistemático das respostas de organismos vivos vem sendo estudado e tais técnicas aplicadas em áreas poluídas (BUSS et al., 2003). No ambiente aquático, plantas, algas, crustáceos, moluscos, peixes, mamíferos, aves e insetos estão entre os mais utilizados no monitoramento biológico (LINS et al., 2010). Entre os invertebrados, destacam-se os macroinvertebrados bentônicos em função do grande número de espécies, do comportamento sedentário, de manterem parte ou todo o ciclo de vida no mesmo local e da sensibilidade de resposta a diferentes tipos e níveis de perturbações (RIBEIRO; UIEDA, 2005).

Esses organismos estão expostos continuamente ao ambiente em que habitam e algumas famílias são amplamente conhecidas por sua tolerância à contaminação, como Chironomidae (CHAGAS et al., 2017). O reconhecimento dessa resposta biótica à qualidade hídrica de ecossistemas deu origem em 1976, na Grã-Bretanha, ao sistema conhecido por Biological Monitoring Working Party Score System (BMWP), um método de biomonitoramento que classifica as famílias de macroinvertebrados aquáticos em grupos, atribuindo uma pontuação referente à sua tolerância às alterações do meio.

A classificação adota um intervalo de 1 a 10, na qual as famílias mais tolerantes à poluição recebem valores menores e as mais sensíveis a essa variação, os maiores (HEPP; RESTELLO, 2007). Após a identificação dos táxons, cada família presente na amostra recebe uma pontuação e se estabelece um somatório geral para cada fragmento coletado, que será compreendido em um intervalo de classes de I a V (BUSS et al., 2003). Cada classe representa um estado da qualidade da água, o que pode significar, em um extremo, águas muito limpas e no outro, fortemente contaminadas.

A ampla distribuição dessas famílias de invertebrados em diferentes padrões climáticos e continentais permite que esse método possa ser utilizado em boa parte do mundo, com algumas adaptações (MONTEIRO et al., 2008). A exemplo do Brasil, a presença em uma amostra de espécies pertencentes à classe Oligochaeta (Famílias Tubificidae e Naididae) e a família Chironomidae, consideradas tolerantes à poluição, indicam ambientes contaminados ou fortemente contaminados (SILVA et al., 2007). Do contrário, a presença e abundância de membros das famílias Ephemeroptera, Plecoptera e Trichoptera 
classificaram o Ribeirão São Bernardo, em Minas Gerais, ausente de alterações ambientais e com a qualidade da água satisfatória (BOAS; CAMARGO, 2017).

Considerando a importância desses macroinvertebrados como indicadores de qualidade ambiental, objetivou-se avaliar um córrego urbano no estado do Pará (Brasil) que recebe o lançamento in natura de esgoto doméstico, disposição inadequada de resíduos sólidos, desmatamento da mata ciliar e ocupações irregulares em sua margem, reflexos da crescente urbanização e fragilidade dos serviços públicos.

\section{METODOLOGIA}

\subsection{LOCAL DO ESTUDO}

O estudo desenvolveu-se no município de Conceição do Araguaia, localizado na porção sudeste do estado do Pará, em que no ano de 2010, apresentava uma população de 45.557 habitantes (IBGE, 2010).

Utilizou-se o córrego urbano Emerêncio como local de pesquisa. Esse manancial apresenta 1,3 km de extensão (da nascente à foz), com uma largura de aproximadamente 10 metros (durante a cheia), apresentando 11 fragmentos vegetacionais, separados por ruas e avenidas (MARTINS et al., 2013; NASCIMENTO; SANTOS, 2012).

Para a classificação e coleta de substratos, adotou-se a metodologia proposta por Silveira et al. (2004) e Callisto (2001) e sugerida no Protocolo de Coleta e Preparação de Amostras de Macroinvertebrados Bentônicos em Riachos. Usualmente, córregos e riachos apresentam quatro classificações de substratos: o folhiço retido em áreas de remanso ou em áreas de correnteza (FC); o folhiço de fundo (FF); a pedra, com detritos vegetais aderidos e/ou perifíton (P) e o sedimento não consolidado (areia, silte e cascalho) (SNC). Dessa forma, coletaram-se três amostras para cada tipo de substrato, de maneira que se pretendeu obter um conjunto de 12 amostras (quatro tipos de substratos com três repetições) em cada ponto de coleta (SILVEIRA et al., 2004).

Como apresentado na Figura 1, distribuíram-se (no sentido da nascente à foz) um total de 11 pontos ou fragmentos, nominalmente sequenciados como E1 ao E11. Realizaram-se cinco campanhas de coletas, sendo as duas primeiras no período chuvoso (abril a maio de 2013) e as três últimas no período seco (julho a setembro de 2013), sendo uma amostragem por mês.

Figura 1 - Pontos amostrados ao longo do córrego Emerêncio, em Conceição do Araguaia/PA.

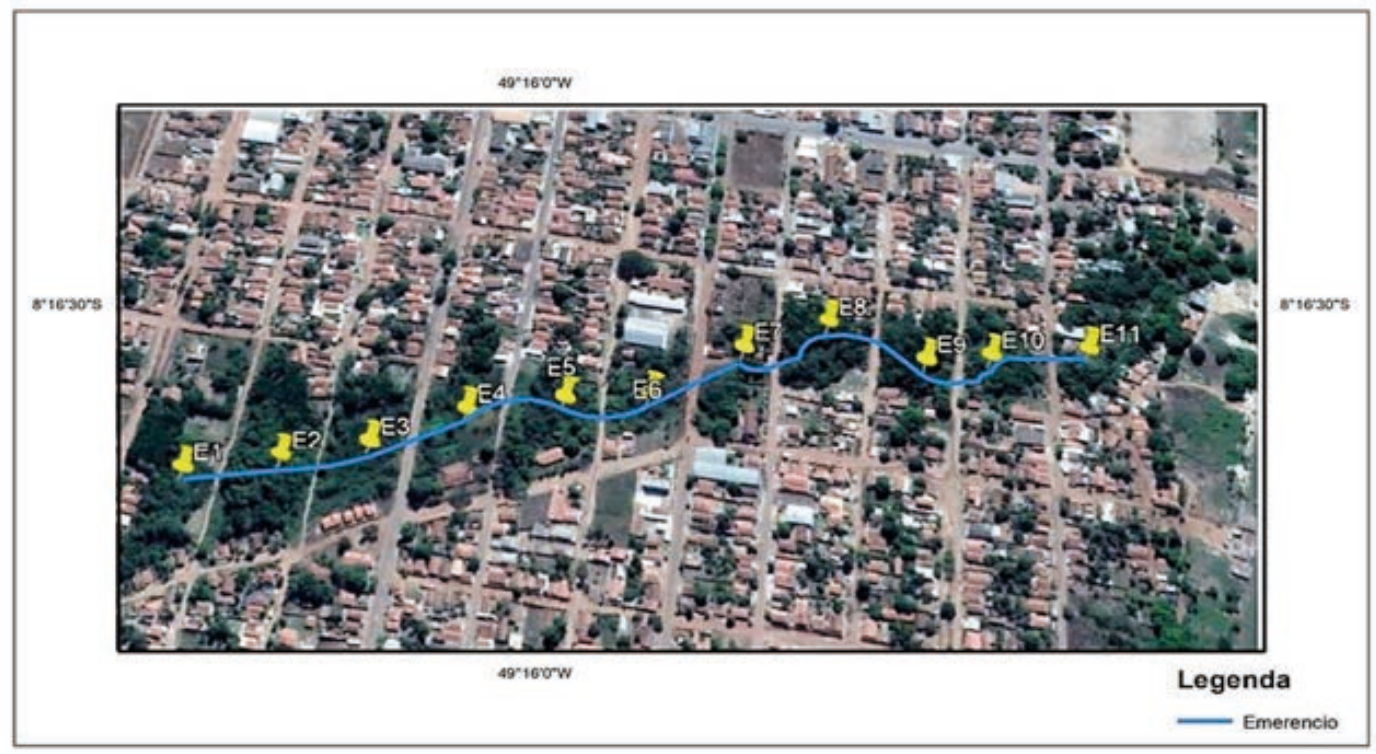

Fonte: Google Earth, 2017. 
A análise biótica seguira as recomendações de Alba-Tercedor (1996), a qual propõe a utilização do índice BMWP como método que atribui determinada pontuação a cada família dos macroinvertebrados verificada para cada fragmento analisado (Tabela 1).

Tabela 1 - Grau de tolerância para os grupos de macroinvertebrados segundo o índice BMWP.

\begin{tabular}{|c|c|c|c|}
\hline CLASSE & VALOR & SIGNIFICADO & COR \\
\hline 1 & $\begin{array}{l}>150 \\
101-120\end{array}$ & $\begin{array}{c}\text { Águas muito limpas } \\
\text { Águas não contaminadas }\end{array}$ & Azul \\
\hline II & $61-100$ & $\begin{array}{l}\text { Águas com alguns efeitos } \\
\text { de contaminação }\end{array}$ & Verde \\
\hline III & $36-60$ & Águas contaminadas & Amarelo \\
\hline IV & $16-35$ & Águas muito contaminadas & Laranja \\
\hline V & $<15$ & $\begin{array}{l}\text { Águas fortemente } \\
\text { contaminadas }\end{array}$ & Roxo \\
\hline
\end{tabular}

Fonte: Alba-Tercedor; Sánchez-Ortega, 1988.

O método BMWP baseia-se na tolerância do impacto ao meio, havendo maior pontuação para as espécies mais sensíveis, e realizando um somatório com aquelas famílias presentes no ambiente, constituindo o índice da qualidade da água local (Tabela 2).

Tabela 2 - Classes de qualidade das águas de acordo com o índice BMWP.

\begin{tabular}{|c|c|}
\hline $\begin{array}{l}\text { Famílias } \\
\end{array}$ & Pontuação \\
\hline $\begin{array}{l}\text { Siphlonuridae, Heptageniidae, Leptophlebiidae, Potamanthidae, } \\
\text { Ephemeridae, } \\
\text { Taeniopterygidae, Leuctridae, Capniidae, Perlodidae, Perlidae, } \\
\text { Chloroperlidae, } \\
\text { Aphelocheiridae, Phryganeidae, Molannidae, Beraeidae, } \\
\text { Odontoceridae, Leptoceridae, Goeridae, Lepidostomatidae, } \\
\text { Brachycentridae, Sericostomatidae, Athericidae, Blephariceridae. }\end{array}$ & 10 \\
\hline $\begin{array}{l}\text { Astacidae Lestidae, Calopterygidae, Gomphidae, } \\
\text { Cordulegasteridae, Aeshnidae, } \\
\text { Corduliidae, Libellulidae, Psychomyiidae, Philopotamidae, } \\
\text { Glossosomatidae. }\end{array}$ & 8 \\
\hline $\begin{array}{l}\text { Ephemerellidae, } \\
\text { Rhyacophilidae, Polycentropodidae, Limnephilidae, Ecnomidae. }\end{array}$ & 7 \\
\hline $\begin{array}{l}\text { Neritidae, Viviparidae, Ancylidae, Thiaridae, Hydroptilidae, } \\
\text { Unionidae, Corophiidae, Gammaridae, Atyidae, Platycnemididae, } \\
\text { Coenagrionidae. }\end{array}$ & 6 \\
\hline $\begin{array}{l}\text { Oligoneuriidae, Polymitarcidae, } \begin{array}{c}\text { Dryopidae, } \\
\text { Helophoridae, Hydrochidae, Hydraenidae, }\end{array} \\
\text { Hydropsychidae, Tipulidae, Simuliidae, } \\
\text { Planariidae, Dendrocoelidae, Dugesiidae. }\end{array}$ & 5 \\
\hline $\begin{array}{l}\text { Baetidae, Caenidae, Haliplidae, Curculionidae, Chrysomelidae, } \\
\text { Tabanidae, Stratiomyidae, Empididae, Dolichopodidae, Dixidae, } \\
\text { Ceratopogonidae, Anthomyidae, Limoniidae, Psychodidae, } \\
\text { Sciomyzidae, Rhagionidae, Sialidae, Piscicolidae, Hidracarina. }\end{array}$ & 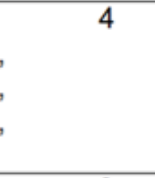 \\
\hline $\begin{array}{l}\text { Mesoveliidae, Hydrometridae, Gerridae, Nepidae, Naucoridae, } \\
\text { Pleidae, Veliidae, } \\
\text { Notonectidae, Corixidae, Helodidae, Hydrophilidae, Hygrobiidae, } \\
\text { Dytiscidae, Gyrinidae, Valvatidae, Hydrobiidae, Lymnaeidae, } \\
\text { Physidae, Planorbidae, Bithyniidae, Bythinellidae, Sphaeridae, } \\
\text { Glossiphoniidae, Hirudidae, Erpobdellidae, Asellidae, Ostracoda. }\end{array}$ & , \\
\hline Chironomidae, Culicidae, Ephydridae, Thaumaleidae. & 2 \\
\hline Oligochaeta (todas as classes), Syrphidae & 1 \\
\hline
\end{tabular}

Fonte: Alba-Tercedor; Sánchez-Ortega (1988). 


\subsection{ATIVIDADES DE CAMPO}

Para a realização das coletas, utilizou-se de um amostrador do tipo Surber, com área útil de $900 \mathrm{~cm}^{2}$ e uma malha coletora de $250 \mu \mathrm{m}$. Este fora posicionado contra a correnteza, e a sua área de amostragem fixada no leito do córrego. Com o auxílio de uma pequena escova, coletou-se parte do substrato para dentro do amostrador.

Transferiu-se o material recolhido para sacos plásticos etiquetados, especificando-se o tipo de substrato, o ponto e a data da respectiva coleta. Em seguida, as amostras foram fixadas em álcool etílico a $70 \%$ e armazenadas no laboratório de ecologia do Instituto Federal do Pará.

\subsection{ATIVIDADES EM LABORATÓRIO}

Em laboratório, realizaram-se os procedimentos de lavagem, flutuação, triagem e identificação dos organismos bentônicos. Nesta etapa, cada material recolhido dos substratos amostrados (folhas, pedras, galhos, perifíton, algas e areia) foi retirado dos sacos plásticos e colocado em um sistema com duas peneiras metálicas ( $25 \mathrm{~cm}$ de diâmetro $\times 10 \mathrm{~cm}$ de altura), e depois lavado em água corrente para retirada do álcool. Em seguida, colocaram-se as amostras em bandejas plásticas com a adição de uma solução supersaturada de açúcar para forçar a flutuação dos organismos e maximizar o processo de triagem.

Após a triagem, os organismos foram dispostos em frascos plásticos transparentes (com capacidade de $3 \mathrm{~mL}$ ), devidamente etiquetados e conservados em álcool a 70\%. A identificação ocorreu a partir de chaves taxonômicas de Angrisano (1995); Azevedo e Hamada (2008); Calor (2007); Epler (2001); Fernández e Domínguez (2001); Lecci e Froehlich (2007); Mariano e Froehlich (2007); Pes et al. (2005); Pinho (2008) e Souza et al. (2007).

\section{RESULTADOS E DISCUSSÕES}

Foi amostrado um total de 2.288 indivíduos que pertenciam aos filos Mollusca, Annelida e Arthropoda, classificados em oito ordens e 23 famílias. No filo Arthropoda, as ordens Díptera e Odonata reuniram juntas o maior número de famílias (61\%), entretanto, da ordem Trichoptera, a família Hydropsychidae foi a mais representativa, com $41 \%$ dos indivíduos (Tabela 3 ). 
Tabela 3 - Distribuição dos Macroinvertebrados Bentônicos nos Grupos Taxonômicos.

\begin{tabular}{|c|c|c|}
\hline Ordem & Família & $\begin{array}{c}N^{\circ} \text { de } \\
\text { Individuos }\end{array}$ \\
\hline \multirow[t]{8}{*}{ Coleóptera } & Hydrophilidae & 03 \\
\hline & Ceratopogonidae & 20 \\
\hline & Chironomidae & 807 \\
\hline & Empididae & 02 \\
\hline & Ephydridae & 4 \\
\hline & Simuliidae & 186 \\
\hline & Tipulidae & 02 \\
\hline & Sciomyzidae & 02 \\
\hline \multirow[t]{2}{*}{ Ephemeroptera } & Ephemeridae & 01 \\
\hline & Caenidae & \\
\hline Megaloptera & Corydalidae & 01 \\
\hline \multirow[t]{4}{*}{ Molusca } & Planorbidae & 05 \\
\hline & Aeshnidae & 01 \\
\hline & Coenagrionidae & 18 \\
\hline & Corduliidae & 02 \\
\hline \multirow[t]{4}{*}{ Odonata } & Gomphidae & 07 \\
\hline & Lestidae & 01 \\
\hline & Libellulidae & 169 \\
\hline & Megapodagrionidae & 03 \\
\hline \multirow[t]{2}{*}{ Annelida } & Oligochaeta & 71 \\
\hline & Ecnomidae & 21 \\
\hline \multirow[t]{2}{*}{ Trichoptera } & Glossosomatidae & 01 \\
\hline & Hydropsychidae & 929 \\
\hline \multicolumn{2}{|l|}{ TOTAL } & 2288 \\
\hline
\end{tabular}

Fonte: Elaboração própria.

No primeiro ponto de coleta (E1), o único substrato encontrado foi do tipo sedimento não compactado e os indivíduos encontrados pertenciam às famílias Chironomidae, Ephydridae e a classe Oligochaeta, indicando a baixa diversidade de espécies (Tabela 4). Segundo Trivinho-Strixino (2011), os organismos da família Chironomidae se alimentam de matéria orgânica depositada no fundo dos rios e lagos, não possuindo nenhuma exigência quanto à diversidade de hábitats, capazes de tolerar ambientes com pouco oxigênio dissolvido, sobrevivendo em condições pouco favoráveis à maior parte dos seres vivos.

Tabela 4 - Ocorrência dos Macroinvertebrados Bentônicos - ponto E1.

\begin{tabular}{|c|c|c|c|c|c|c|}
\hline \multicolumn{7}{|c|}{ Mês da Coleta } \\
\hline Substrato & $04 / 13$ & $05 / 13$ & $07 / 13$ & $08 / 13$ & $09 / 13$ & Total \\
\hline \multicolumn{7}{|c|}{ Grupos taxonômicos e pontuação BMWP } \\
\hline SNC & $\begin{array}{c}\text { Chironomidae } \\
\text { (2), } \\
\text { Ephydridae } \\
(2)\end{array}$ & - & - & $\begin{array}{l}\text { Oligochaeta } \\
\text { (5) }\end{array}$ & - & 5 \\
\hline
\end{tabular}


O somatório das famílias (5) demonstrou que no índice BMWP a água desse ponto é classificada como Classe V, ou seja, fortemente contaminada (Tabela 4). Nesse fragmento, o córrego é afetado pelo escoamento de sedimentos misturados às águas pluviais e de esgoto, principalmente durante o período chuvoso, ocasionando o assoreamento e a descarga de efluentes domésticos provenientes das residências construídas a menos de dez metros de distância do córrego. Nessas ocasiões, costuma-se haver o risco de redução do oxigênio dissolvido, afetando diretamente a fauna aquática presente no local (MORAES; JORDÃO, 2002).

Contrariamente ao anterior, o ponto E2 é um dos fragmentos onde se observa a conservação e permanência da vegetação nativa, com árvores de grande porte e ausência de deslizamentos nas encostas. No local foram encontrados os quatro tipos de substratos descritos, favorecendo o aparecimento de famílias características de ambientes sem alteração, como Coenagrionidae, Libellulidae e Hydropsychidae (Tabela 5). Para Callisto et al. (2001), ambientes com alta diversidade de hábitats contêm maior riqueza taxonômica em relação àqueles com baixa diversidade de hábitats.

Segundo Siqueira e Henry-Silva (2011), a manutenção de um gradiente ecológico, onde a composição do ambiente está mais próxima de sua formação original, pode ser responsável pelo contínuo fluvial, ou seja, nesse trecho a adaptação dos organismos bentônicos às condições físicas e ao período de maior entrada de matéria orgânica particulada (chuvoso), provavelmente pode ser um dos aspectos que justificam a riqueza encontrada.

Tabela 5 - Ocorrência dos Macroinvertebrados Bentônicos - ponto E2.

\begin{tabular}{ccccccc}
\hline \multicolumn{5}{c}{ Mês da Coleta } \\
\hline Substrato & $04 / 13$ & $05 / 13$ & $07 / 13$ & $08 / 13$ & $09 / 13$ & Total \\
\hline \multicolumn{7}{c}{ Grupos taxonômicos e pontuação BMWP } \\
\hline FC & Chironomidae & Chironomidae & Chironomidae & Chironomidae & 55 \\
FF & Libellulidae & & $(2)$, & $(2)$, & $(2)$ & \\
SNC & $(8)$ & Hydropsychidae & Hydropsychidae & & \\
& & & $(5)$ & $(5)$ & & \\
\hline
\end{tabular}

Fonte: Elaboração própria

Mesmo apresentando alta diversidade, o somatório resultante do índice BMWP no ponto E2 (55) indica uma classificação de águas contaminadas, o que pode ser explicado pela variação sazonal da riqueza, com muitas famílias ocorrendo em um único mês no período chuvoso e a predominância de uma a duas famílias nos meses seguintes, pelo tamanho do fragmento e pela descarga de efluentes a montante (ponto E1). A família Chironomidae estava marcadamente presente em todos os meses amostrados, e a inclusão de manilhas de concreto nas porções do córrego interceptadas por ruas, que mantêm em alguns locais águas mais correntes e, em outros, de maior remanso, pode ser um fator de influência, dado o hábito generalista dos membros dessa família, segundo Sanseverino e Nessimian (2001).

No ponto E3 (Tabela 6), o uso agrícola fez com que parte do curso do córrego fosse desviado para abastecer tanques de piscicultura, mantendo porções com grande volume de água. A vegetação transita de espécies arbóreas para capim alto, indicando o desmatamento e o aparecimento de espécies exóticas invasoras. Segundo Esteves (2011), a perda da vegetação de margens de rios acaba por influenciar na textura e composição de seus substratos, um conceito que pode explicar o predomínio de SNC nesse ponto, onde apenas organismos da família Ephemeridae foram encontrados, contribuindo para que a pontuação do índice BMWP equivalesse a dez, o que classificou suas águas como fortemente contaminadas. 
Tabela 6 - Ocorrência dos Macroinvertebrados Bentônicos - ponto E3.

\begin{tabular}{ccccccc}
\hline \multicolumn{7}{c}{ Mês da Coleta } \\
\hline Substrato & $04 / 13$ & $05 / 13$ & $07 / 13$ & $08 / 13$ & $09 / 13$ & Total \\
\hline & - & Grupos taxonômicos e pontuação BMWP & \\
\hline SNC & - & - & - & - & Ephemeridae & 10
\end{tabular}

Fonte: Elaboração própria.

A disposição de resíduos sólidos está presente em quase todos os fragmentos do córrego Emerêncio, indicando a deficiência nos serviços de limpeza urbana e a carência de educação ambiental da população. Uma pesquisa desenvolvida por Santos et al. (2011) revelou que 67\% dos entrevistados residentes no bairro próximo ao córrego classificam o serviço de coleta de lixo como ruim, fator que pode ocasionar o descarte inadequado de resíduos domiciliares.

Já no ponto E4 (Tabela 7), as perturbações ambientais estão relacionadas à disposição de resíduos sólidos no leito do rio e ao assoreamento, provocado pelo escoamento superficial das ruas e perda da vegetação nativa, com predominância de capim alto e, consequentemente, homogeneização de hábitat, sendo encontrado apenas o substrato do tipo SNC.

Tabela 7 - Ocorrência dos Macroinvertebrados Bentônicos - ponto E4.

\begin{tabular}{|c|c|c|c|c|c|c|}
\hline \multicolumn{7}{|c|}{ Mês da Coleta } \\
\hline Substrato & $04 / 13$ & $05 / 13$ & $07 / 13$ & $08 / 13$ & $09 / 13$ & Total \\
\hline \multicolumn{7}{|c|}{ Grupos taxonômicos e pontuação BMWP } \\
\hline SNC & $\begin{array}{c}\text { Ephydridae } \\
\text { (2) }\end{array}$ & $\begin{array}{l}\text { Oligochaeta } \\
\text { (1) }\end{array}$ & $\begin{array}{l}\text { Oligochaeta } \\
\text { (1), }\end{array}$ & $\begin{array}{l}\text { Oligochaeta } \\
\text { (1) }\end{array}$ & - & 8 \\
\hline
\end{tabular}

Planorbidae

(3)

Fonte: Elaboração própria.

Observa-se na Tabela 7 que as famílias dos macroinvertebrados encontrados neste fragmento têm pontuações baixas no índice BMWP, com variações na presença e ausência dos organismos ao longo dos meses do ano, indicando um ambiente fortemente contaminado (8, Classe V). No local, encontraramse organismos tolerantes, como indivíduos da classe Oligochaeta que podem viver em condições de anoxia e não apresentam nenhum tipo de exigência quanto à diversidade de hábitats e micro-hábitats (GOULART; CALLISTO, 2003). As transformações ambientais caracterizadas ainda no ponto E3 e em seguida no ponto E4 justificam essas observações, a exemplo do registrado por Barrilli et al. (2012) sobre a ocupação do solo às margens do Rio Monjolinho, como a urbanização e as atividades inerentes à agricultura e pecuária, que refletiram no desaparecimento de espécies de macroinvertebrados bentônicos sensíveis e promoveram a dominância de espécies resistentes, como os táxons Oligochaeta e Chironomidae.

O ponto E5 foi o que apresentou maior vulnerabilidade ao assoreamento, por se encontrar em uma região mais baixa e ao final de um declive, com encostas desprotegidas e interceptado por ruas não pavimentadas, fazendo com que muito do sedimento carreado pelo escoamento superficial (pluvial e de efluentes) adentrasse ao córrego. Árvores de pequeno porte compõem a mata ciliar desse local, sendo que os substratos característicos verificados foram FC e SNC (Tabela 8). Assim como nos pontos anteriores, o índice biótico BMWP apresentou uma baixa pontuação (14, Classe V), classificando-se a qualidade da água desse fragmento como fortemente contaminada. 
Tabela 8 - Ocorrência dos Macroinvertebrados Bentônicos - ponto E5

\begin{tabular}{|c|c|c|c|c|c|c|}
\hline \multicolumn{7}{|c|}{ Mês da Coleta } \\
\hline Substrato & $04 / 13$ & $05 / 13$ & $07 / 13$ & $08 / 13$ & $09 / 13$ & Total \\
\hline \multicolumn{7}{|c|}{ Grupos taxonômicos e pontuação BMWP } \\
\hline $\begin{array}{l}\text { FC } \\
\text { SNC }\end{array}$ & $\begin{array}{c}\text { Chironomidae } \\
\text { (2), } \\
\text { Tipulidae } \\
\text { (5) }\end{array}$ & $\begin{array}{c}\text { Chironomidae } \\
\text { (2) }\end{array}$ & - & - & $\begin{array}{c}\text { Corydalidae } \\
\text { (4), } \\
\text { Oligochaeta } \\
\text { (1) }\end{array}$ & 14 \\
\hline
\end{tabular}

Fonte: Elaboração própria.

O fragmento que corresponde ao ponto E6 se apresenta como uma região brejosa, cuja vegetação é composta por pequenas plantas e árvores, com águas mais calmas. Nesse local foi possível observar uma maior diversidade no período chuvoso, fato já registrado no ponto E2, que pode estar relacionado às modificações ambientais causadas pelas cheias. Ambientes com característica de brejo são quase sempre homogêneos quanto às condições topográficas e composição biótica (IVANAUSKAS et al., 1997) e devido aos aspectos de uso do córrego já mencionado em pontos a montante, há o favorecimento da presença de famílias mais tolerantes, gerando assim um índice biótico BMWP onde se classificam as águas como muito contaminadas (21, Classe IV).

Tabela 9 - Ocorrência dos Macroinvertebrados Bentônicos - ponto E6.

\begin{tabular}{|c|c|c|c|c|c|c|}
\hline \multicolumn{7}{|c|}{ Mês da Coleta } \\
\hline Substrato & $04 / 13$ & $05 / 13$ & $07 / 13$ & $08 / 13$ & $09 / 13$ & Total \\
\hline \multicolumn{7}{|c|}{ Grupos taxonômicos e pontuação BMWP } \\
\hline SNC & $\begin{array}{c}\text { Chironomidae } \\
\text { (2) }\end{array}$ & $\begin{array}{c}\text { Chironomidae } \\
\text { (2), } \\
\text { Libellulidae } \\
(8), \\
\text { Caenidae } \\
\text { (5) }\end{array}$ & $\begin{array}{c}\text { Planorbidae } \\
\text { (3) }\end{array}$ & - & $\begin{array}{c}\text { Oligochaeta } \\
\text { (1) }\end{array}$ & 55 \\
\hline
\end{tabular}

Fonte: Elaboração própria.

No ponto E7 (Tabela 10) são verificados substratos de dois tipos, o SNC e FC, indicando que nesse trecho já existem materiais suficientes para a criação de refúgios e a presença de uma corrente hídrica para melhorar os aspectos químicos e físicos que, segundo Fantin-Cruz et al. (2011), a velocidade da corrente atua sobre a temperatura de pequenos córregos, que influi na solubilidade de compostos químicos, nas reações bioquímicas, estratificando a biota aquática e influenciando a composição de espécies. Esse trecho apresentou uma boa representatividade de famílias indicadoras de ambientes limpos durante todo o período de amostragem, sugerindo uma solubilização ou contenção da descarga de efluentes que pode ter ocorrido no trecho anterior (E6), dado que matas brejosas são reconhecidas por atuarem como um filtro biológico (POTT; POTT, 2002). 
Tabela 10 - Ocorrência dos Macroinvertebrados Bentônicos - ponto E7

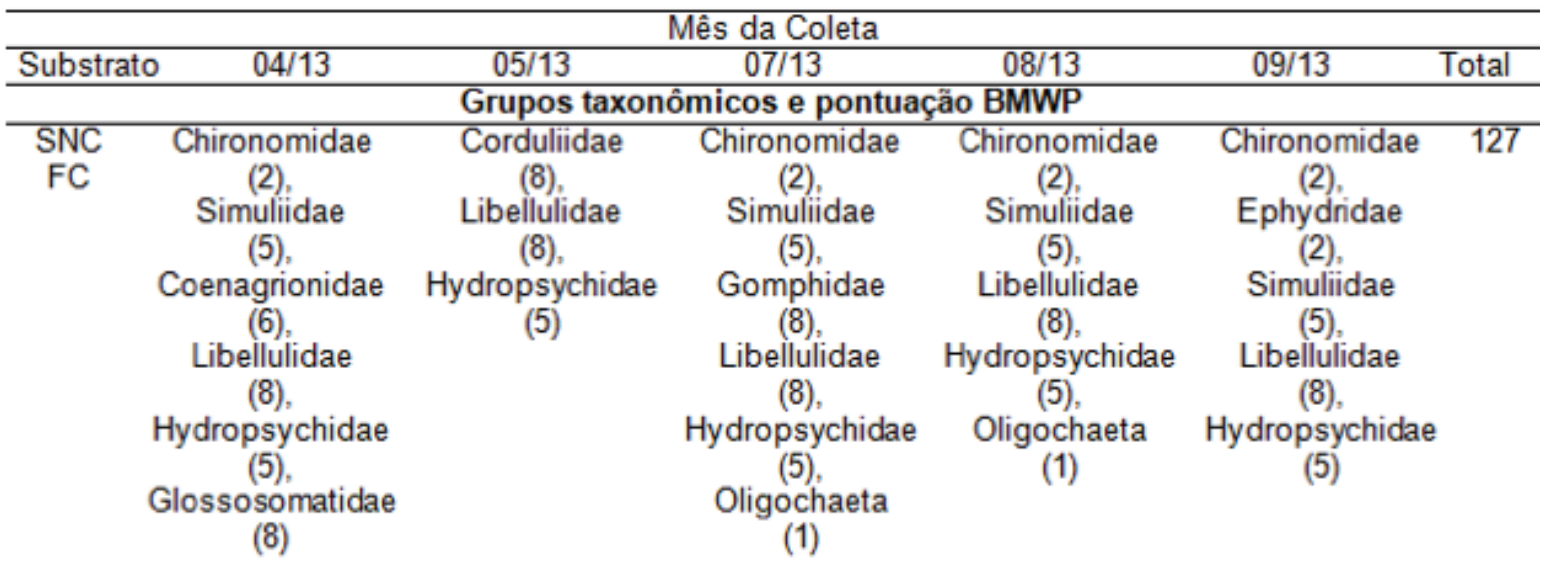

Fonte: Elaboração própria.

Entre as famílias descritas no ponto E7, destaca-se a Hydropsychidae (Tabela 10) por ser a mais representativa em número de indivíduos no córrego. Segundo Esteves (2011), essa família apresenta distribuição seletiva quanto ao tipo de substrato, uma vez que seleciona materiais de qualidade para a adequação de seu hábitat particular. De acordo com a referência do índice biótico, esse ponto foi classificado com águas muito limpas e/ou águas não contaminadas. Contudo, verifica-se que a mata ciliar está desaparecendo do local, em decorrência da construção de residências próximas ao leito e, consequentemente, poderá trazer no futuro o lançamento de resíduos sólidos em sua margem.

A representatividade dos macroinvertebrados bentônicos se manteve constante no ponto E8 (Tabela 11) durante todo o período amostrado, com exceção do mês de setembro, quando nenhum indivíduo foi coletado. O local possui áreas de represamento e a mata ciliar encontra-se bastante alterada, constituída por pequenas árvores, com algumas espécies exóticas (Bambusa vulgaris, Syzygium cumini, Mangifera indica) e herbáceas. Encontraram-se os substratos do tipo SNC e FC, o que já explica a presença de grande diversidade de famílias, entretanto, as modificações antrópicas exercem já alguns efeitos sobre o córrego, pois no índice BMWP suas águas são classificadas como Classe II, indicando alguns efeitos de contaminação.

Tabela 11 - Ocorrência dos Macroinvertebrados Bentônicos - ponto E8.

\begin{tabular}{|c|c|c|c|c|c|c|}
\hline \multicolumn{7}{|c|}{ Mês da Coleta } \\
\hline Substrato & $04 / 13$ & $05 / 13$ & $07 / 13$ & $08 / 13$ & $09 / 13$ & Total \\
\hline \multicolumn{7}{|c|}{ Grupos taxonômicos e pontuação BMWP } \\
\hline $\begin{array}{c}\text { SNC } \\
\text { FC }\end{array}$ & $\begin{array}{c}\text { Chironomidae } \\
\text { (2), } \\
\text { Simuliidae } \\
\text { (5), } \\
\text { Coenagrionidae } \\
\text { (6), }\end{array}$ & $\begin{array}{c}\text { Coenagrionidae } \\
(6), \\
\text { Libellulidae } \\
(8)\end{array}$ & $\begin{array}{c}\text { Chironomidae } \\
(2), \\
\text { Simuliidae } \\
(5), \\
\text { Libellulidae } \\
\text { (8) }\end{array}$ & $\begin{array}{c}\text { Chironomidae } \\
\text { (2), } \\
\text { Simuliidae } \\
\text { (5), } \\
\text { Libellulidae } \\
\text { (8) }\end{array}$ & - & 65 \\
\hline
\end{tabular}

(8) 
No ponto E9 os substratos encontrados foram dos tipos SNC, P e FC e na vegetação da margem predomina o açaí (Euterpe oleracea), uma espécie nativa comum das várzeas amazônicas, além da mata ciliar se apresentar com bom aspecto de conservação e ausência de resíduos sólidos no local. Novamente foi observada a conservação das condições originais do rio e esse contínuo fluvial pode ser explicado pela ocorrência representativa das famílias indicadoras de águas não contaminadas, tais como: Megapodagrionidae (10), Libellulidae (8), Lestidae (8), Aeshnidae (8) e Gomphidae (8) (Tabela 12).

Tabela 12 - Ocorrência dos Macroinvertebrados Bentônicos - ponto E9

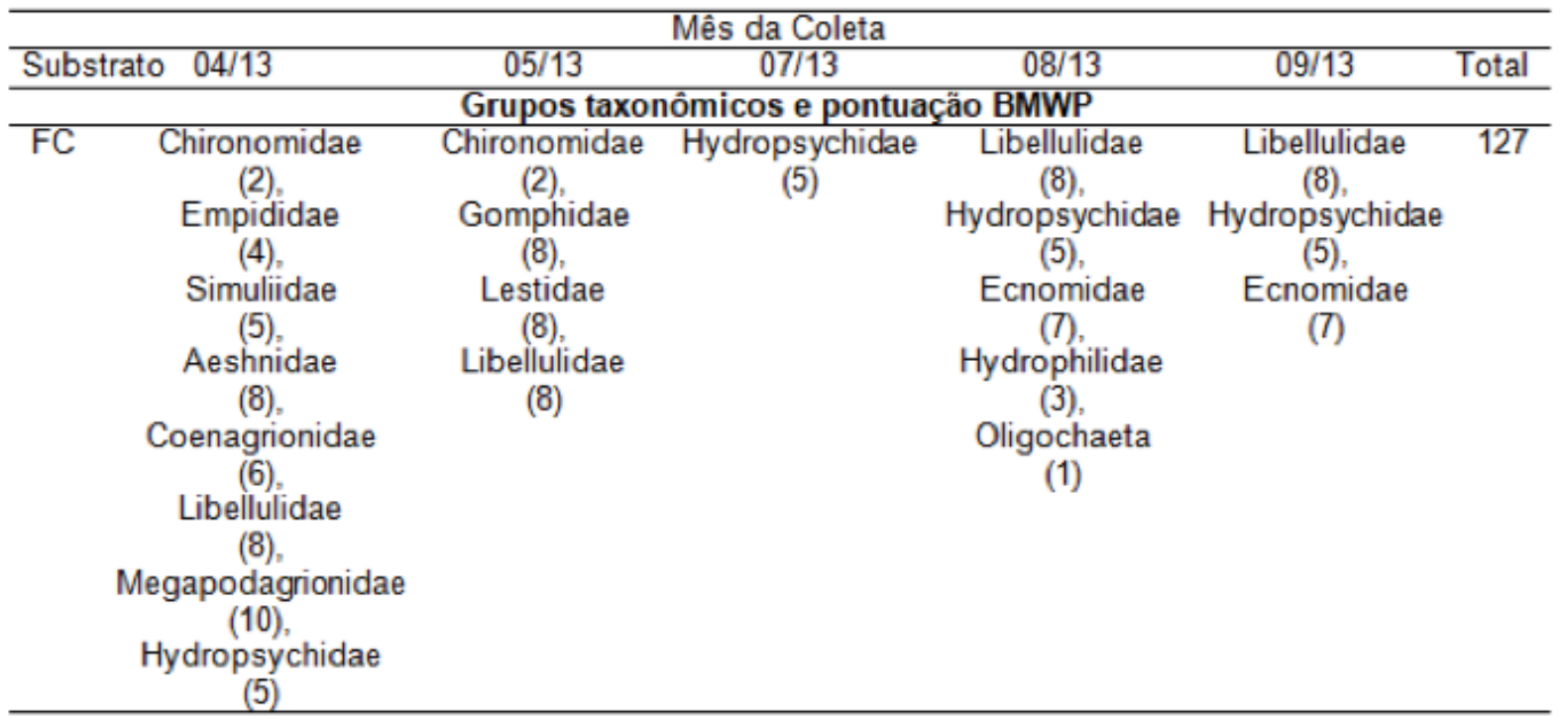

Fonte: Elaboração própria.

Em contraste ao ponto amostral anterior (E9), o fragmento E10 apresentou uma condição ambiental homogênea, com apenas um tipo de substrato, o SNC e representatividade apenas da família Chironomidae e da classe Oligochaeta nos dois meses correspondentes ao período chuvoso (abril e maio). Dada a ocorrência de famílias indicadoras de ambientes contaminados e, portanto, de baixos valores no índice, as águas desse local foram classificadas como fortemente contaminadas (Tabela 13).

Tabela 13 - Ocorrência dos Macroinvertebrados Bentônicos - ponto E10

\begin{tabular}{|c|c|c|c|c|c|c|}
\hline \multicolumn{7}{|c|}{ Mês da Coleta } \\
\hline Substrato & $04 / 13$ & $05 / 13$ & $07 / 13$ & $08 / 13$ & $09 / 13$ & Total \\
\hline \multicolumn{7}{|c|}{ Grupos taxonômicos e pontuação BMWP } \\
\hline SNC & $\begin{array}{c}\text { Chironomidae } \\
\text { (2) }\end{array}$ & $\begin{array}{l}\text { Oligochaeta } \\
\text { (1) }\end{array}$ & - & - & - & 3 \\
\hline
\end{tabular}

Fonte: Elaboração própria.

Nesse ponto, a presença de grande quantidade de resíduos sólidos descartados inadequadamente pode ser um fator que contribui para o processo de contaminação hídrica. Em um levantamento realizado por Martins et al. (2013) com os moradores que habitam às margens do córrego Emerêncio, $29 \%$ afirmaram que descartam seu lixo no curso de água e justificam suas ações por não haver no município coleta regular de lixo. Outro aspecto que pode contribuir para a contaminação do curso hídrico é a reduzida oferta de tratamento de esgotos domésticos, que, segundo registros do IBGE (disponível em: https://cidades.ibge.gov.br), são tratados adequadamente apenas 4,8\% do esgoto em todo o município. 
O último ponto amostrado (E11), que já se encontra na foz do córrego e próximo ao encontro com o Rio Araguaia, está localizado no bairro conhecido por vila dos pescadores e nesse trecho a tubulação de esgoto das residências construídas às margens se encontrava exposta para o córrego, realizando regularmente o lançamento de seus efluentes domésticos diretamente no curso hídrico, bem como de seus resíduos sólidos, que eram depositados nas encostas. Os substratos encontrados nesse ponto foram o SNC e P (Tabela 14), nos quais foram encontradas famílias indicadoras de águas fortemente contaminadas (Classe V).

Tabela 14 - Ocorrência dos Macroinvertebrados Bentônicos - ponto E11.

\begin{tabular}{|c|c|c|c|c|c|c|}
\hline \multicolumn{7}{|c|}{ Mês da Coleta } \\
\hline Substrato & $04 / 13$ & $05 / 13$ & $07 / 13$ & $08 / 13$ & $09 / 13$ & Total \\
\hline \multicolumn{7}{|c|}{ Grupos taxonômicos e pontuação BMWP } \\
\hline $\mathrm{SNC}$ & $\begin{array}{c}\text { Chironomidae } \\
\text { (2) }\end{array}$ & $\begin{array}{c}\text { Coenagrionidae } \\
\text { (6), } \\
\text { Oligochaeta } \\
\text { (1) }\end{array}$ & - & $\begin{array}{l}\text { Empididae } \\
\text { (4), } \\
\text { Oligochaeta } \\
\text { (1) }\end{array}$ & - & 14 \\
\hline
\end{tabular}

Fonte: Elaboração própria.

\section{CONCLUSÕES}

De maneira geral, a análise da comunidade dos macroinvertebrados bentônicos demonstrou a relevância desses organismos como bioindicadores da qualidade da água em ecossistemas aquáticos. O uso do índice biótico BMWP como uma ferramenta de classificação se mostrou satisfatório para o córrego Emerêncio quando, no local, foram observadas alterações visíveis, como desmatamento, disposição inadequada de resíduos sólidos e lançamento de efluentes domésticos sem tratamento, que são instrumentalmente difíceis de mensurar quando se consideram todos os aspectos que envolvem a transformação completa e o desaparecimento de tais alterações no ambiente.

Verificou-se que a composição de táxons está amplamente relacionada aos tipos de substratos e ao respectivo grau de conservação da mata ciliar, uma vez que nos pontos onde havia a predominância de um único substrato e a presença de alterações antrópicas, normalmente havia a ocorrência marcante de famílias tolerantes. Outro aspecto relevante é a sensibilidade dos organismos para responderem tanto à continuidade quanto à descontinuidade de um gradiente ecológico, considerando o pequeno tamanho do córrego observado e a distância entre os pontos de amostragem, o que reforça a importância da mata na manutenção dos hábitats, contenção de encostas e como um filtro de substâncias nocivas.

O biomonitoramento mostrou-se uma ferramenta útil, simples e de baixo custo, pois não são necessários equipamentos de medição precisa e insumos laboratoriais de alto custo, tornando-se uma alternativa promissora para avaliações rápidas e demonstrações de caráter informativo para o público em geral. Entretanto, o método se torna bem mais efetivo quando aplicado em estudos de longo prazo, considerando a sazonalidade, os aspectos físico-químicos, topográficos, geológicos, de uso e conservação do recurso.

Estudos que determinam a contaminação das águas, como a mensurada nos diversos pontos do córrego Emerêncio, bem como as características ambientais observadas nos respectivos fragmentos (mata ciliar, descarga de efluentes domésticos, presença de resíduos sólidos e falta de pavimentação das ruas), indicam a deficiência na gestão municipal do planejamento urbano, o descaso público com a conservação de corpos hídricos e na manutenção de áreas verdes, tão necessárias à melhoria da qualidade de vida nas cidades. 


\section{REFERÊNCIAS}

ALBA-TERCEDOR, J. Macroinvertebrados acuáticos y calidade de las aguas de los rios. IV SIMPOSIO DEL AGUA EM ANDULACIA (SIAGA). Anais... Almeria, v. 2, p. 203-213, 1996.

ALBA-TERCEDOR, J.; SÁNCHEZ-ORTEGA, A. Un método rápido y simples para evaluar la calidad biológica de las aguas corrientes basado en el Hellawell. (1978). Limnética 4, p. 51-56, 1988.

ANGRISANO, E. B. Insecta Trichoptera. In: LOPRETTO, E. C.; TELL, G. (Ed.). Ecossistemas de águas continentales: metodologias para su estúdio. La Plata: EdicionesSur. v. 3, p. 1199-1237, 1995.

AZEVEDO, C. A. S.; HAMADA, N. Megaloptera. In: Guia on-line: identificação de larvas de insetos aquáticos do estado de São Paulo. Disponível em: <http://sites.ffclrp.usp.br/aguadoce/guiaonline>. Acesso em: 15 out. 2017.

BARRILLI, G. H. C.; ROCHA, O.; DE LUCCA, J. V. Avaliação da qualidade da água nos córregos Fazzari e Monjolinho no campus da UFSCar. Periódico Eletrônico Fórum Ambiental da Alta Paulista, v. 8, n. 2, 2012.

BOAS, A. H. V.; CAMARGO, F. V. Avaliação rápida da qualidade da água utilizando invertebrados bentônicos, através dos índices bióticos BMWP' e ASPT no Ribeirão São Bernardo, Piranguçu, Sul de Minas Gerais. CES Revista, v. 31, n. 1, p. 7-25, 2017.

BUSS, D. F.; BAPTISTA, D. F.; NESSIMIAN, J. L. Bases conceituais para a aplicação de biomonitoramento em programas de avaliação da qualidade da água de rios. Cadernos de Saúde Pública. v. 19, n. 2, p. 465-473, 2003.

CALLISTO, M.; MORETTI, M.; GOULART, M. Macroinvertebrados bentônicos como ferramenta para avaliar a saúde de riachos. Revista Brasileira de Recursos Hídricos, v. 6, n. 1, p. 71-82, 2001.

CALOR, A. R. Trichoptera. In: Guia on-line de Identificação de larvas de Insetos Aquáticos do Estado de São Paulo. Disponível em: <http://sites.ffclrp.usp.br/aguadoce/index_trico>. Acesso em: 20 nov. 2017.

CHAGAS, F. B. et al. Utilização da estrutura de comunidades de macroinvertebrados bentônicos como indicador de qualidade da água em rios no sul do Brasil. Revista Ambiente e Água. v. 12, n. 3, p. 416425, 2017.

EPLER, J. H. Identification manual for the larval Chironomidae (Diptera) of Florida. Tallahassee: Department of Environmental Protection. Division of Water Facilities, 319 p., 2001.

ESTEVES, F. A. Fundamentos de Limnologia. 3. ed. Rio de Janeiro: Interciência, p. 581-597, 2011.

FANTIN-CRUZ, I.; TONDATO, K. K.; MOTTA-MARQUES, D. Regime térmico em águas correntes e sua importância na estrutura do hábitat e na biologia de organismos aquáticos. Caminhos de Geografia, v. 11, n. 36, 2010.

FERNÁNDEZ, H. R.; DOMÍNGUEZ, E. Guía para la determinacíon de losartrópodos bentônicos sudamericanos. Tucumán: Editorial Universitario de Tucumán. 282 p., 2001.

GONÇALVES, F. B. Análise comparativa de índices bióticos de avaliação de qualidade de água, utilizando macroinvertebrados, em um rio litorâneo do Estado do Paraná. Dissertação (Mestrado) Universidade Federal do Paraná. 2009.

GOULART, M. D.; CALLISTO, M. Bioindicadores de qualidade de água como ferramenta em estudos de impacto ambiental. Revista da Fapam, v. 2, n. 1, p. 153-164, 2003.

HEPP, L. U.; RESTELLO, R. M. Macroinvertebrados bentônicos como bioindicadores da qualidade das águas do Alto Uruguai Gaúcho. Edifapes, Erechim, p. 75-86, 2007. 
INSTITUTO BRASILEIRO DE GEOGRAFIA E ESTATÍSTICA. Disponível em: <www.ibge.gov.br>. Acesso em: 10 out. 2016.

IVANAUSKAS, N. M.; RODRIGUES, R. R.; NAVE, A. G. Aspectos ecológicos de um trecho de floresta de brejo em Itatinga, SP: florística, fitossociologia e seletividade de espécies. Brazilian Journal of Botany, v. 20, n. 2, p. 139-153, 1997.

LECCI, L. S.; FROEHLICH, C. G. Plecoptera. In: Guia on-line: identificação de larvas de insetos aquáticos do estado de São Paulo. Disponível em: <http://sites.ffclrp.usp.br/aguadoce/guiaonline> Acesso em: 14 set. 2017.

LIMA, B. P.; MAMEDE, G. L.; NETO, I. E. L. Monitoramento e modelagem da qualidade de água em uma bacia hidrográfica semiárida. Eng. sanit. ambient, v. 23, n. 1, p. 125-135, 2018.

LINS, J. A. P. N. et al. Uso de peixes como biomarcadores para monitoramento ambiental aquático. Rev. Acad. Ciên. Agrár. Ambient, v. 8, p. 469-484, 2010.

MARIANO, R.; FROEHLICH, C. G. Ephemeroptera. In: Guia on-line: identificação de larvas de insetos aquáticos do estado de São Paulo. Disponível em: <http://sites.ffclrp.usp.br/aguadoce/guiaonline>. Acesso em: 12 mar. 2017.

MARTINS, D. E. M. et al. Percepção Ambiental dos moradores residentes na área de preservação permanente do córrego Emerêncio no município de Conceição do Araguaia-PA. IV CONGRESSO BRASILEIRO DE GESTÃO AMBIENTAL, Salvador-BA, 2013.

MONTEIRO, T. R.; OLIVEIRA, L. G.; GODOY, B. S. Biomonitoramento da qualidade de água utilizando macroinvertebrados bentônicos: adaptação do índice biótico BMWP à bacia do Rio Meia Ponte-GO. Oecol. Bras., v. 12, n. 3, p. 553-563, 2008.

MORAES, D. S. L.; JORDÃO, B. Q. O. Degradação de Recursos Hídricos e seus efeitos sobre a saúde humana. Revista Saúde Pública, v. 36, n. 3, 2002.

NASCIMENTO, T. P. A.; SANTOS, M. L. Diagnóstico das condições florísticas das margens do Emerêncio, afluente do Rio Araguaia no sul do Pará. Biodiversidade, v. 11, n.1, 2012.

PES, A. M. O.; HAMADA, N.; NESSIMIAN, J. L. Chaves de identificação de larvas para famílias e gêneros de Trichoptera (Insecta) da Amazônia Central, Brasil. Revista Brasileira de Entomologia, v. 49, n. 2, p. 181-204, 2005.

PINHO, L. C. Diptera. In: Guia on-line: identificação de larvas de insetos aquáticos do estado de São Paulo. Disponível em: <http://sites.ffclrp.usp.br/aguadoce/guiaonline> Acesso em: 16 abr. 2017.

POTT, V. J.; POTT, A. Potencial de uso de plantas aquáticas na despoluição da água. Embrapa Gado de Corte, 2002.

RIBEIRO, L. O.; UIEDA, V. S. Estrutura da comunidade de macroinvertebrados bentônicos de um riacho de serra em Itatinga, São Paulo, Brasil. Revista Brasileira de Zoologia, v. 22, p. 613-618, 2005.

SANSEVERINO, A. M.; NESSIMIAN, J. L. Hábitats de larvas de Chironomidae (Insecta, Diptera) em riachos de Mata Atlântica no Estado do Rio de Janeiro. Acta Limnologica Brasiliensia, v. 13, n. 1, p. 29-38, 2001.

SANTOS, L. B.; CORREIA, D. L. S.; SANTOS J. C. Macroinvertebrados bentônicos como bioindicadores do impacto urbano sobre o Rio Uberaba (MG). Journal of Environmental Analysis and Progress, v. 1, n. 1, p. 34-42, 2016.

SANTOS, M. L. et al. Levantamento das condições do saneamento básico no bairro Emerêncio e Jardim Petrópolis do município de Conceição do Araguaia - PA. In: II CONGRESSO BRASILEIRO DE GESTÃO AMBIENTAL. Londrina, 2011. 
SILVA, F. L. et al. Desempenho de dois índices biológicos na avaliação da qualidade das águas do Córrego Vargem Limpa, Bauru, SP, através de macroinvertebrados bentônicos. Pan-American Journal of Aquatic Sciences, v. 2, n. 3, p. 231-234, 2007.

SILVA, N. T. C. Macroinvertebrados bentônicos em áreas com diferentes graus de preservação ambiental na Bacia do Ribeirão Mestre d'Armas, DF. Dissertação (Mestrado em Ecologia) - Universidade de Brasília. 99 p. 2007.

SILVEIRA, M. P.; QUEIROZ, J. F.; BOEIRA, R. C. Protocolo de Coleta e Preparação de Amostras de Macroinvertebrados Bentônicos em Riachos. Jaguariúna: Emprapa. 7 p. (Comunicado Técnico 19), 2004.

SOUZA, L. O. I.; COSTA, J. M.; OLDRINI, B. B. Odonata. In: Guia on-line: identificação de larvas de insetos aquáticos do estado de São Paulo. Disponível em: <http://sites.ffclrp.usp.br/aguadoce/Guia_online>. Acesso em: 24 abr. 2017.

TRIVINHO-STRIXINO, S. Larvas de Chironomidae: guia de identificação. São Carlos, UFSCar. 371 p, 2011. 